\title{
Design of a Silicon Overlay Glass Waveguide Sensor
}

\author{
Kristian E. Medri, Robert C. Gauthier \\ Department of Electronics, Carleton University, Ottawa, Canada \\ E-mail:kmedri@doe.carleton.ca \\ Received August 13, 2011; revised September 12, 2011; accepted September 24, 2011
}

\begin{abstract}
A glass based slab waveguide, coated with a thin patterned high dielectric overlay, is configured into a refractive index sensor. The asymmetric nature of the waveguide configuration is exploited by keeping the mode in the slab waveguide while enhancing the field level in the overlay-superstrate. The sensor's response is examined using the FDTD simulation technique. A sensitivity of up to one part in $10^{5}$ in the index of refraction discrimination is determined. The nature of the sensor ensures optical fibre compatibility, requires sub- $\mu \mathrm{L}$ sample volumes and provides a high resolution.
\end{abstract}

Keywords: Refractive Index Sensor, Slab Waveguide, Patterned High Dielectric Overlay, FDTD, Integrated Optics

\section{Introduction}

Silica based planar lightwave circuits (PLCs) evolved from simple passive waveguide structures to complex active devices containing resonators and filters.[1,2] When interfaced with optical fibers, PLCs can demonstrate low insertion losses due to the compatibility of the materials, large modal overlap and relaxed manufacturing tolerances. In general, glass based integrated optics demonstrate a slow response when configured as active devices and are large due to the limited dielectric contrast between guiding and substrate-superstrate layers. In the near and distant future it is expected that the optical fiber will continue to dominate as the signal carrying medium for optical telecommunications and sensing. The inter-compatibility requirements of active and passive integrated-optic devices and the optical fibre technology will remain. This will require low loss, low insertion loss, and fast IO components. These requirements can be met by a glass based waveguide design as the light guiding region, to minimize fibre coupling and insertion losses, then adding a thin high dielectric overlay of patterned silicon at the waveguide superstrate interface to retain the high dielectric contrast and response speed. This results in a hybrid platform of silica and silicon akin to a strip loaded waveguide.

Refractive index sensors can be used for many applications. Previously proposed sensors which have similar footprints and responsivities include a 13 um sensor providing $90 \mathrm{~nm} / \mathrm{RIU}[3]$ and an 8 um sensor providing 130
nm/RIU [4] which have small non fiber compatible mode sizes. The researchers of the former include a comparison to alternative sensing structures while the latter mentions a much longer 173 um sensor providing a lower resolution of $33 \mathrm{~nm} / \mathrm{RIU}$ [5]. This longer sensor has a larger mode size. They mention the option of using a 10 um plasmon sensor providing $465 \mathrm{~nm} / \mathrm{RIU}$ but with a large $10 \mathrm{~dB}$ loss.

In order to demonstrate the principles involved in this hybrid waveguide configuration we employ a slab waveguide in glass and a silicon overlay patterned as a Bragg grating [6]. The functionality is explored by examining the structure's performance as an index of refraction sensor sensitized to the sensing region comprised of the superstrate region above the grating as well as in the etched regions of the grating. The next section presents the design of the sensor and discusses the modeling technique employed. The sensor's performance is then presented.

\section{Design}

The simplest planar optical structure is the traditional three layer glass slab waveguide (superstrate-waveguide -substrate), modified here into a 4 layer structure by adding a thin high dielectric overlay at the superstratewaveguide interface (superstrate-overlay-wave-guidesubstrate). The basic theory and analytical solution for the three layer structure is widely described $[7,8]$ and has been extended to address multilayer structures with di- 
electric overlays $[9,10]$. This enables the modal profiles and propagation constants for the three layer and four layer structures to be determined with high accuracy. However, when the overlay is patterned in the direction of light propagation, the perturbation approach [11] to the waveguide analysis does not apply as the dielectric contrast is very high between waveguide and overlay layers. The optical properties of such structures can be modeled using numerical methods such as the FiniteDifference-Time-Domain (FDTD) [12,13] technique provided that the thin overlay layer is well resolved in the discretized grid.

The structure under consideration is shown in Figure 1. The substrate layer is glass with an index of refraction $\mathrm{n}_{4}=1.500$ and extends to $-\infty$ in the $\mathrm{x}$ direction. The waveguide layer is formed from a $1 \mu \mathrm{m}$ thick slab of index $n_{3}=1.600$. The superstrate layer is typically air with an index of $n_{1}=1.000$ and extends to $+\infty$. The thin overlay, shown as a dashed line, has an index of $n_{2}=$ 3.480, a thickness $t_{2}=17.5 \mathrm{~nm}$ and is patterned as a Bragg grating in the $\mathrm{z}$ direction. The sensing region is considered to be that of the superstrate in the grating region including the etched regions of the grating. The grating pitch is designed to meet the Bragg condition for a wavelength of $1550 \mathrm{~nm}$ when the sensing region contains the highest index material to be sensed. Also for this material, the thickness $t_{2}$ of the overlay is chosen such that the overall waveguiding structure remains single mode with the maximum value of the effective index below 1.600 for the wavelengths considered. The alternating high and low effective index regions of the $40 \mu \mathrm{m}$ long grating are each made a quarter wavelength in length which is determined by using the propagation constants of the 3 and 4 layer modal solutions [14].

\section{Simulation and Discussion}

A 2-D FDTD environment was set up to model the propagation of light in the waveguide sensor structure. The $(\mathrm{x}, \mathrm{z})$ plane of a simulation configuration is shown in Figure 1 and consists of a 3-layer segment of $1 \mu \mathrm{m}$ for the source plus $12 \mu \mathrm{m}$ followed by a $40 \mu \mathrm{m}$ long grating and terminated by a $12 \mu \mathrm{m} 3$-layer waveguide. The entire domain was bordered by a $1 \mu \mathrm{m}$ PML with matched dielectric values. The y-direction was taken as infinite and out of the page. The entire structure was discretized on a

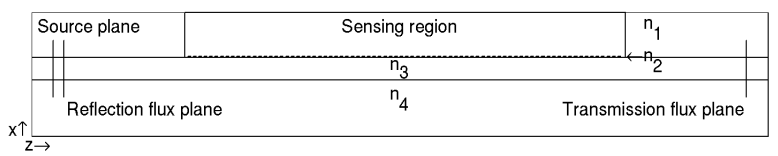

Figure 1. Slab waveguide in glass with thin patterned silicon grating overlay. Source, reflection and transmission planes used in FDTD simulation shown. square grid at 160 points per micron resolution to ensure suitable structure resolution and minimal numerical dispersion as confirmed through convergence tests. The input source consisted of a short time duration pulse with a profile and field components matched to those for the 3-layer geometry obtained by solving the field equation for the field polarized in the y direction $\left(E_{Y}\right)$. The multiwavelength source is defined as the discrete-time derivative of a Gaussian,

$$
E_{y}^{\prime}=(-i \omega)^{-1} \frac{\partial}{\partial t} \exp \left(-i \omega t-\left(t-t_{0}\right)^{2} / 2 w^{2}\right)
$$

with a central wavelength of $1550 \mathrm{~nm}$ and $\mathrm{w}=0.334$. The source plane was spaced $12 \mu \mathrm{m}$ from the grating input and the detector planes are located $11 \mu \mathrm{m}$ from the grating end. The power reflectance and transmittance, normalized to source output, were computed on each detector plane following the technique presented in [15].

The simulated spectrums for this structure when pure water is in the sensing region, $\mathrm{n}_{1}=1.315$ for $25^{\circ} \mathrm{C}$ [16], are shown in Figure 2. In the transmission spectrum the deepest dip at just under $1550 \mathrm{~nm}$ corresponds to the Bragg wavelength of the grating and the secondary dip at a lower wavelength is associated with power coupling to substrate and radiation modes. A band pass region for wavelengths beyond the Bragg wavelength is also observed. The reflection spectrum is a dual of the transmission spectrum. In the analysis of the sensor the transmission spectrums are examined and attention is directed at the Bragg dip's wavelength and depth dependence on the sensing region's index.

The structure's response with pure water in the sensing region is used as a reference for the response with the index of the sensing region increased to a maximum of $n_{1}=1.465$ (a change of 0.15 ) in steps of 0.025 . The index values and range were chosen to be representative of the wavelengths, temperature, and solutes considered. The highest refractive index of 1.465 corresponds to a $77 \%$,
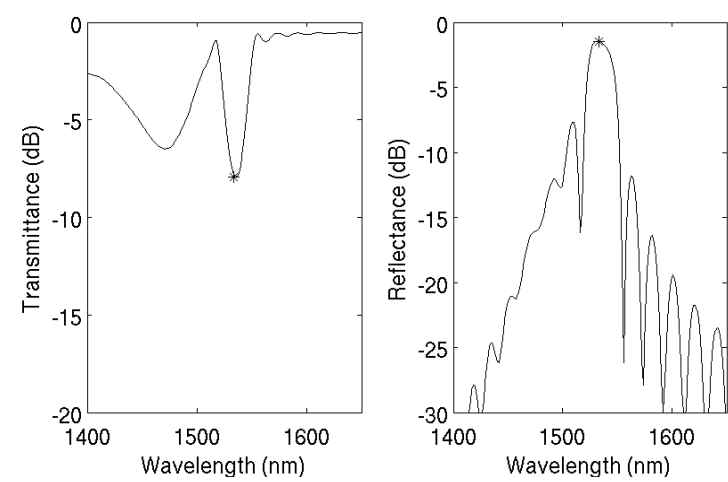

Figure 2. Transmittance and reflectance spectrums for the sensor with pure water in the sensing region. Bragg wavelength denoted by star. 
The highest refractive index of 1.465 corresponds to a $77 \%$, or 77 Brix [17], sucrose solution given a refractive index shift similar to that of water due to change of wavelength and the few degree temperature change from the $20^{\circ} \mathrm{C}$ data [18]. As the refractive index in the sensing region is increased the Bragg dip shifts to a longer wavelength as shown in Figure 3. In addition, the increasing index has the effect of modifying the grating's duty cycle and modal overlap between the cascade of three and 4 layer waveguide regions making up the grating. The grating being designed for the maximum index in the sensing region ensures that the Bragg dip gets monotonically deeper over the sensing range of index values.

The sensor's response can be evaluated using two criteria. The first is to track the change in the Bragg dip wavelength and the second is to monitor the change in signal strength for a fixed operation wavelength. The sensor's performance in both modes of operation can be determined from the family of traces in Figure 3. In order to consider the use of the shift in the Bragg dip versus refractive index, the locations of the minimums in the primary dip are marked with stars in Figure 3. A wavelength shift is $85 \mathrm{~nm} / \mathrm{RIU}$ is determined and assuming a wavelength resolution of 1 pm [19] a refractive index resolution of $10^{-5}$ is possible.

When the output intensity is monitored directly, it is instructive to use the pure water trace as a reference and then determine the change in output intensity as a function of wavelength and sensing region index relative to the chosen reference. The families of traces in Figure 4 represent change in output intensity derived from the data of Figure 3. The maximum of the change in transmittance is used to start the sensor wavelength selection.

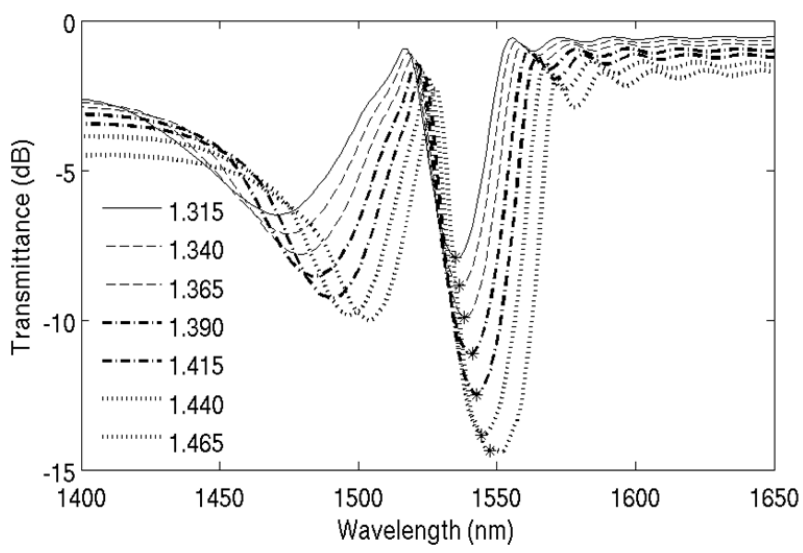

Figure 3. Transmittance for different superstrate refractive indices. The solid line represents the pure water trace with $n_{1}=1.315$, while two each of the dashed, dashed and dotted, and dotted lines represent $n_{1}=1.340,1.365,1.390,1.415$, 1.440 , and 1.465 respectively. Star points on each trace indicate the location of the Bragg wavelength of the grating.
Next the response for wavelengths slightly above and below this were compared to find a wavelength with the strongest change in response matching the center of the index range considered.

The sensor response for a wavelength of $1556 \mathrm{~nm}$ is shown in Figure 5. Depending on the desired sensing index range, the sensing wavelength can be optimized for the maximum slope and linearity for that range. Since amplitude detection units are able to resolve $0.1 \%$ intensity changes [20], this provides a sensitivity of $1.8 \times 10^{-4}$ for a $40 \mu \mathrm{m}$ sensor. Fabrication roughness can reduce the sharpness and strength of a grating dip which can be compensated by increased length [21].

\section{Conclusions}

A refractive index sensor has been presented based on the optical properties of a slab waveguide coated with a patterned thin high dielectric overlay. The hybrid nature of the entire waveguide configuration makes it possible to keep the waveguide mode in the slab waveguide compatible to optical fiber while enhancing the evanescent

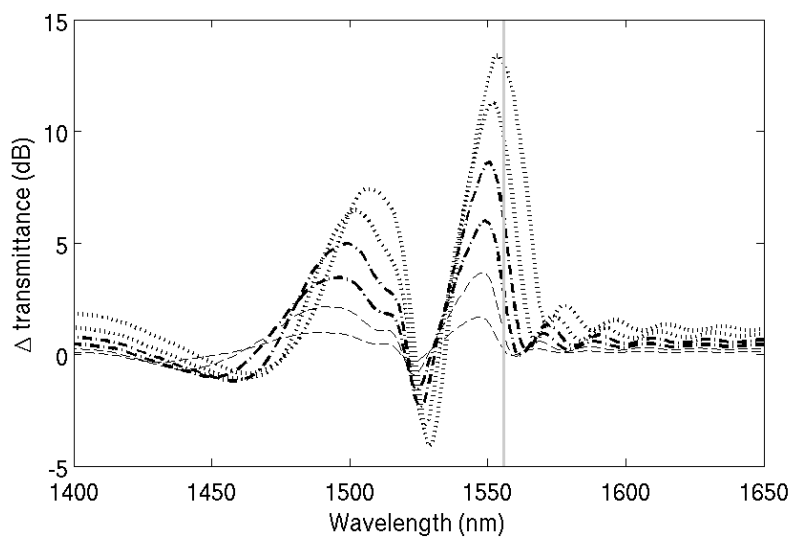

Figure 4. Change in transmittance. Two each of the dashed, dashed and dotted, and dotted lines represent $n_{1}=1.340$, $1.365,1.390,1.415,1.440$, and 1.465 versus the $n_{1}=1.315$ simulation respectively.

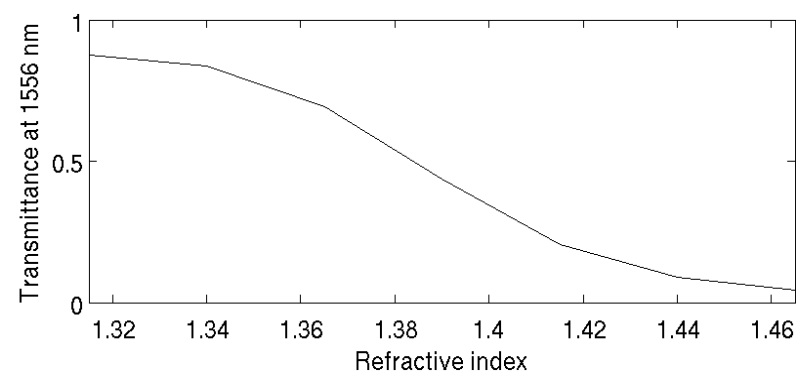

Figure 5. Sensor normalized power response for source wavelength of $1556 \mathrm{~nm}$. 
wave-overlay interaction using the high dielectric.The sensitivity of the guided light to variations in the cladding refractive index was examined using FDTD simulations and transmission spectrums are obtained for various superstrate refractive indices. The response of the sensor is examined using both the change in the Bragg dip wavelength and transmitted signal strength and is shown to provide an index resolution of up to one part in $10^{5}$.

\section{Acknowledgements}

The authors gratefully acknowledge NSERC, CIPI, and the reviewers for their support.

\section{References}

[1] M. Kawachi, "Recent Progress in Silica-Based Planar Lightwave Circuits on Silicon,” IEE Proceedings: Optoelectronics, Vol. 143, No. 5, 1996, pp. 257-262. doi:10.1049/ip-opt:19960493

[2] J. Broquin, "Glass Integrated Optics: State of the Art and Position toward Other Technologies," Proceedings of SPIE, Vol. 6475, No. 7, 2007, pp. 1-13. doi:10.1117/12.706785

[3] P. Prabhathan, V. M. Murukeshan and J. Zhang, "Compact SOI Nanowire Refractive Index Sensor Using Phase Shifted Bragg Grating,” Optics Express, Vol. 17, No. 17, 2009, pp. 15330-15341. doi:10.1364/OE.17.015330

[4] S. Mandal, R. Akhmechet, L. Chen, S. Nugen, A. Baeumner and D. Erickson, "Nanoscale Optofluidic Sensor Arrays for Dengue Virus Detection," Nanoengineering: Fabrication, Properties, Optics and Devices IV, The International Society for Optical Engineering (SPIE), 2007.

[5] V. M. N. Passaro, R. Loiacono, G. D’Amico and F. De Leonardis, "Design of Bragg Grating Sensors Based on Submicrometer Optical Rib Waveguides in SOI," IEEE Sensors Journal, Vol. 8, No. 9, 2008, pp. 1603-1611. doi:10.1109/JSEN.2008.929068

[6] K. E. Medri and R. C. Gauthier, "Patterned Overlays: Thin Silicon Layer Applied to Glass Waveguides," Proceedings of SPIE, Vol. 7943, No. L, 2011, pp. 1-12. doi:10.1117/12.873426

[7] K. Okamoto, "Fundamentals of Optical Waveguides," Second Edition. Academic Press, Cleveland, 2005.

[8] C. Chen, "Foundations for Guided-Wave Optics," John Wiley, Hoboken, 2006. doi:10.1002/0470042222

[9] C. Chen, "Development and Implementation of Novel Numerical Techniques for Integrated Optics and Microwave Planar Structures," Ottawa-Carleton Institute for Electrical and Computer Engineering, Ottawa, 2000.
[10] A. Yimit, A. G. Rossberg, T. Amemiya and K. Itoh, "Thin Film Composite Optical Waveguides for Sensor Applications: A Review,” Talanta, Vol. 65, No. 5, 2005, pp. 1102-1109. doi:10.1016/j.talanta.2004.06.045

[11] A. Yariv, "Coupled-Mode Theory for Guided-Wave Optics,” IEEE Journal of Quantum Electronics, Vol. 9, No. 9, 1973, pp. 919-933. doi:10.1109/JQE.1973.1077767

[12] A. F. Oskooi, D. Roundy, M. Ibanescu, P. Bermel, J. D. Joannopoulos and S. G. Johnson, "Meep: A Flexible Free-Software Package for Electromagnetic Simulations by the FDTD Method," Computer Physics Communications, Vol. 181, No. 2, 2010, pp. 687-702. doi:10.1016/j.cpc.2009.11.008

[13] I. Giuntoni, M. Krause, H. Renner, J. Bruns, A. Gajda, E. Brinkmeyer and K. Petermann, "Numerical Survey on Bragg Reflectors in Silicon-On-Insulator Waveguides," 5th International Conference on Group IV Photonics, 17-19September 2008, pp. 285-287.

[14] M. Hammer and O. V. Ivanova, "Effective Index Approximations of Photonic Crystal Slabs: A 2-to-1-D Assessment," Optical and Quantum Electronics, Vol. 41, No. 4, 2009, pp. 267-283. doi:10.1007/s11082-009-9349-3

[15] B. E. A. Saleh and M. C. Teich, "Fundamentals of Photonics,” John Wiley, Hoboken, 1991. doi:10.1002/0471213748

[16] A. H. Harvey, J. S. Gallagher and J. M. H. L. Sengers, "Revised Formulation for the Refractive Index of Water and Steam as a Function of Wavelength, Temperature and Density," Journal of Physical and Chemical Reference Data, Vol. 27, No. 7, 1998, pp. 761-74.

[17] F.J. Bates, et al., "Polarimetry, Saccharimetry and the Sugars," National Bureau of Standards C440, U.S. Government Printing Office, Wahington, DC, 1942.

[18] R. C. Weast, "Handbook of Chemistry and Physics," CRC Press, Cleveland, 1977.

[19] A. Iadicicco, A. Cusano, A. Cutolo, R. Bernini and M. Giordano, “Thinned Fiber Bragg Gratings as High Sensitivity Refractive Index Sensor," IEEE Photonics Technology Letters, Vol. 16, No. 4, 2004, pp. 1149-1151. doi:10.1109/LPT.2004.824972

[20] A. Iadicicco, S. Campopiano, A. Cutolo, M. Giordano and A. Cusano, "Refractive Index Sensor Based on Microstructured Fiber Bragg Grating," IEEE Photonics Technology Letters, Vol. 17, No. 6, 2005, pp. 1250-1252. doi:10.1109/LPT.2005.846570

[21] L. Poladian, F. Ladouceur and P. D. Miller, "Effects of Surface Roughness on Gratings," Journal of the Optical Society of America B (Optical Physics), Vol. 14, No. 6, 1997, pp. 1339-1344. 